\title{
COMPARISON OF ACTIVE CONTROL TECHNIQUES OVER A DIHEDRAL PLANE
}

\author{
Emmanuel Creusé 1,2
}

\begin{abstract}
This work is devoted to the numerical comparison of four active control techniques in order to increase the pressure recovery generated by the deceleration of a slightly compressible viscous flow over a dihedral plane. It is performed by the use of vortex generator jets and intrusive sensors. The governing equations, the two-dimensional direct numerical simulation code and the flow configuration are first briefly recalled. Then, the objective of the control is carefully displayed, and the uncontrolled flow described. The main part of this work deals with the explanation, the implementation and the comparison of four active control strategies: closed loop control, adaptative control, physical ramp control and sub-optimal control. Each of these techniques is of different nature, and results are very formative to understand what is important - or less - to make the control efficient.
\end{abstract}

Mathematics Subject Classification. 93C95, 76N25, 76F40, 76G25.

Received February 26, 2001.

\section{INTRODUCTION}

Nowadays, control in fluid mechanics represents a very important research axis. Delaying the detachment of a boundary layer, or reducing the drag on a profile are for example crucial industrial and economic objectives. Besides, a lot of papers, congresses and workshops are devoted to this theme.

On the one hand, a lot of processes are passive control ones. By passive control, we mean that the actions generated on the system are time independant. The idea can be to optimize the shape or to work on the material of a profile on which the fluid evolves, in order to improve a desired property. A reference work in fluid mechanics was for example devoted to the direct numerical simulation of a turbulent flow over riblets, to decrease the drag coefficient [8]. Indeed, passive control is most of the time simple to carry out and rather cheap. Nevertheless, it is determinated once and for all. An alteration in the nature of the flow can so make it inefficient, or even damaging.

On the other hand, the second possibility is to resort to active control. By active control, we mean that the actions on the system are not only time dependant, but also function of the flow evolution itself. A very large number of experiences and studies, both experimentally and numerically have been realized for the last decade. A very complete inventory was drawed up by Moin and Bewley before 1994 [22], as well as by Hernandez

Keywords and phrases: Active control, compressible viscous flow, subsonic evolution.

1 M.A.C.S., Université de Valenciennes, le Mont Houy, 59313 Valenciennes Cedex 9, France;

e-mail: ecreuse@univ-valenciennes.fr

2 M.A.B., Université Bordeaux 1, 33405 Talence, France. 
before 1996 [16]. Several more recent references will be mentionned throughout this paper, on which the work developped below is based.

The object of this paper is to compare four active control techniques on a simple configuration, corresponding to the evolution of a slightly compressible viscous flow over a dihedral plane for a laminar evolution. This configuration was already studied. First, relevant boundary conditions were found to ensure a physically coherent simulation [7], and then the dynamics of the uncontrolled flow was carefully described [13]. The four different active control techniques have been chosen among different horizons, to understand as far as possible the advantages and drawbacks of each approach. We will deal here with mechanical active control, statistical active control, physical ramp control, and mathematical active control. Whatever the control studied, the control device is always composed of one or several Vortex Generator Jets, to blow or to suck up fluid through the dihedral, and intrusive sensors.

The paper is decomposed like explained here. The first part shortly recalls the governing equations and the numerical scheme used to solve them, before displaying the flow configuration. In the second part, the purpose of the control is explained, and the uncontrolled simulation presented. Then, in the third part, each of the four active control techniques is developped, and for each of them the results obtained on the dihedral configuration are presented, compared and commented.

\section{Governing EQUATIONS AND NUMERICAL CODE}

\subsection{Governing equations}

The governing equations are the well known two-dimensional compressible Navier-Stokes equations, given in their non-dimensionalized formulation in an orthonormal system and with usual notations by:

$$
\begin{cases}\frac{\partial U}{\partial t}+\nabla \cdot F(U) & =\frac{1}{R e} \nabla \cdot G(U, \nabla U) \\ U(t=0) & =U_{0},\end{cases}
$$

with:

$$
U=[\rho, \rho u, \rho v, \rho E]^{T}
$$

Here, the total energy per unit of mass is denoted $E$, and $R e$ is the numerical Reynolds number. The convective flux are given by:

$$
\begin{aligned}
& F_{x}(U)=\left[\rho u, \rho u^{2}+p, \rho u v,(\rho E+p) u\right]^{T}, \\
& F_{y}(U)=\left[\rho v, \rho u v, \rho v^{2}+p,(\rho E+p) v\right]^{T} .
\end{aligned}
$$

The diffusive ones are given by:

$$
\begin{aligned}
& G_{x}(U, \nabla U)=\left[0, \sigma_{x x}, \sigma_{x y}, \beta_{x}\right]^{T}, \\
& G_{y}(U, \nabla U)=\left[0, \sigma_{x y}, \sigma_{y y}, \beta_{y}\right]^{T},
\end{aligned}
$$


with:

$$
\begin{aligned}
& \beta_{x}=u \sigma_{x x}+v \sigma_{x y}+\frac{\gamma k}{\operatorname{Pr}} \frac{\partial T}{\partial x}, \\
& \beta_{y}=u \sigma_{x y}+v \sigma_{y y}+\frac{\gamma k}{\operatorname{Pr}} \frac{\partial T}{\partial y},
\end{aligned}
$$

where $T$ is the temperature, $\gamma=1.4, k=1, \operatorname{Pr}=0.72$, and:

$$
\begin{aligned}
\sigma_{x x} & =\frac{4}{3} \frac{\partial u}{\partial x}-\frac{2}{3} \frac{\partial v}{\partial y} \\
\sigma_{y y} & =\frac{4}{3} \frac{\partial v}{\partial y}-\frac{2}{3} \frac{\partial u}{\partial x} \\
\sigma_{x y} & =\sigma_{y x}=\frac{\partial u}{\partial y}+\frac{\partial v}{\partial x} .
\end{aligned}
$$

The system is then closed by the state equation:

$$
p=(\gamma-1) \rho T
$$

as well as by the relation:

$$
\rho E=\frac{1}{2} \rho\|\vec{u}\|^{2}+\frac{p}{\gamma-1}
$$

\subsection{Numerical code}

The previous equations are numerically solved by a direct numerical simulation on an unstructured mesh of triangular cells. The convective term approximation is made with a finite volume method, with Roe solver and a MUSCL technique in order to bring the second order accuracy in space. No flux limiter is needed, since the solutions considered here do not exhibit discontinuities. The diffusive term approximation is ensured by a finite element method, with classical conform $P_{1}$ elements. The centered process also brings the second order accuracy in space. Finally, the temporal integration of the residuals is performed with the Heun method, by using a mass-lumping procedure. The temporal integration is so an explicit one, and a CFL condition has to be respected to ensure the numerical stability of the scheme. More details about the numerical scheme will be found for example in [7] or [14].

\subsection{Flow configuration}

The flow considered in this work is the two-dimensional evolution of a viscous compressible flow over a dihedral plane. The computational domain is displayed in dashed lines in Figure 1. The height of the boundary layer at point $\mathrm{A}$, on the inflow boundary (a), is denoted $\delta$. The horizontal velocity very far from the dihedral is denoted $U_{\infty}$, and $\mu$ and $\rho_{r}$ are respectively the dynamic viscosity coefficient and a characteristic density of the fluid. A characteristic Reynolds number of the flow $R e_{\delta}$ can then be deduced from these previous values:

$$
\operatorname{Re}_{\delta}=\frac{\rho_{r} U_{\infty} \delta}{\mu}
$$




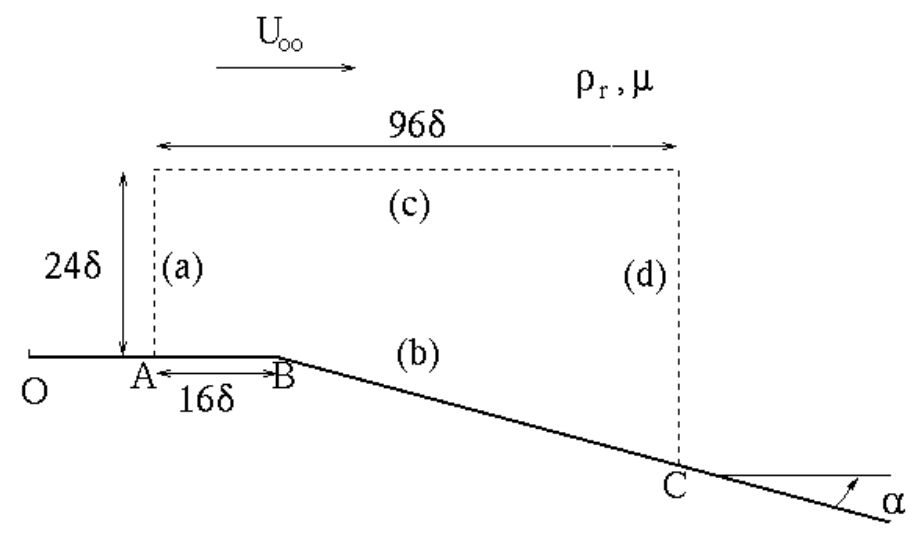

Figure 1. Computational domain.

It should be noted that the distance between the leading edge $\mathrm{O}$ and the point $\mathrm{A}$ is completely defined as a function of $\delta$ and $R e_{\delta}$. Indeed, by using the Blasius approximation for an incompressible laminar boundary layer [11], we have:

$$
\delta \approx \frac{5 \sqrt{\mu} \sqrt{O A}}{\sqrt{\rho_{r} U_{\infty}}} .
$$

From (1) and (2), we get:

$$
O A \approx \frac{\delta R e_{\delta}}{25} .
$$

In this work, $R e_{\delta}=400$. Thus, the flow is in a laminar evolution. Mach number at infinity is $M=0.2$, which corresponds to a slightly compressible flow. The size of the computational domain is defined as a function of $\delta$, as indicated in Figure 1. $\alpha$ is equal to $10^{\circ}$. The grid is composed of $201 \times 101$ nodes. To obtain a good resolution of the boundary layer and the separation point instabilities, the grid distribution is compacted near the dihedral wall regions in the normal direction to the boundary and horizontally in the vicinity of the point B. For the value $R e_{\delta}=400,20$ nodes are located in the height of the boundary layer at the entry of the domain.

\subsection{Boundary conditions}

The boundary (b) corresponds to an isothermal no-slip wall, and the boundaries (a, c) and (d) are artificial ones, without any physical reality. On each of these frontiers, we have to specify relevant boundary conditions. On the (a) boundary, a subsonic inflow condition is used. The two components of the velocity $u_{i n}$ and $v_{i n}$ (adimensionalized by $U_{\infty}$ and corresponding to the Blasius profile) as well as the temperature $T_{0}$ are strongly specified:

$$
\left\{\begin{array}{l}
\eta=\frac{5 y}{\delta}, \quad T_{i n}=T_{0} \\
u_{i n}=f^{\prime}(\eta), \quad v_{i n}=\frac{5}{2 \operatorname{Re} \delta}\left(\eta f^{\prime}(\eta)-f(\eta)\right),
\end{array}\right.
$$

where $f(\eta)$ and $f^{\prime}(\eta)$ are tabulated functions of $\eta$ [11]. The pressure is then deduced with the continuity equation. On the (b) boundary, an isothermal no-slip wall is used. The two components of the velocity are set to zero, and the temperature $T_{0}$ is strongly specified. The pressure is also deduced with the continuity equation. On the (c) boundary, we use the non reflecting boundary condition with a pressure recall, in order to specify to the flow the static pressure at infinity. For a very accurate description of the implementation of the $(\mathrm{a}, \mathrm{b})$ and 
(c) boundary conditions, see [24]. Finally, on the (d) boundary, we use a recent artificial subsonic boundary condition, which allows us to specify the value of the unique entering characteristic wave's amplitude in a good way with regard to the physics of the problem (see [7]).

\section{Control Purpose AND DESCRIPtion of The UnCONTROLlED FlOW}

\subsection{Control purpose}

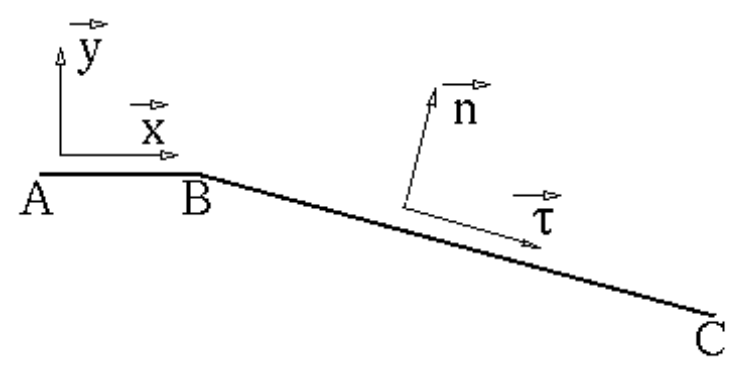

FiguRE 2. $\vec{\tau}$ and $\vec{n}$ definitions.

Let us note $\vec{\tau}$ and $\vec{n}$ respectively the tangent and the normal vectors to the dihedral between the point $\mathrm{B}$ and the point $\mathrm{C}$ (Fig. 2). $u_{\tau}$ (respectively $u_{n}$ ) is the velocity in the $\vec{\tau}$ direction (respectively in the $\vec{n}$ direction). In such a configuration, the total force induced by the flow on the upper side of the part $\mathrm{BC}$ of the dihedral can be mainly defined as the sum of a pressure force and of a friction one:

$$
\vec{F}=\int_{[B C]}-p \vec{n}+\mu \frac{\partial u_{\tau}}{\partial n} \vec{\tau} \mathrm{d} l .
$$

The upward thrust, defined as the component in the $\vec{x}$ direction of the force $\vec{F}$, is so given by:

$$
T=\int_{[B C]}-p \sin \alpha+\mu \frac{\partial u_{\tau}}{\partial n} \cos \alpha \mathrm{d} l \quad(\alpha>0) .
$$

For a laminar evolution, we will consider that the pressure force is predominant with regard to the friction one, and $T$ will be in a first approximation defined as:

$$
T \approx \int_{[B C]}-p \sin \alpha \mathrm{d} l
$$

The objective of the control procedure is to maximize this approximated upward thrust. In order to do it, the $T$ value is compared to an ideal value $T_{i d}$, which would have been obtained in the case of an incompressible perfect inviscid fluid on the same configuration ( $\rho$ is then considered as constant). For such a flow, we note respectively $U$ and $p$ the average velocity and the average pressure on the current section whose height is given by $S$ (Fig. 3). The inflow average velocity and pressure, as well as the height of the inflow section are respectively denoted $U_{e}$, $p_{e}$ and $S_{e}$. Provided that the top artificial boundary is high enough to ensure that the velocity of the fluid is nearly horizontal on this boundary, we can write the mass conservation principle:

$$
U S=U_{e} S_{e}
$$




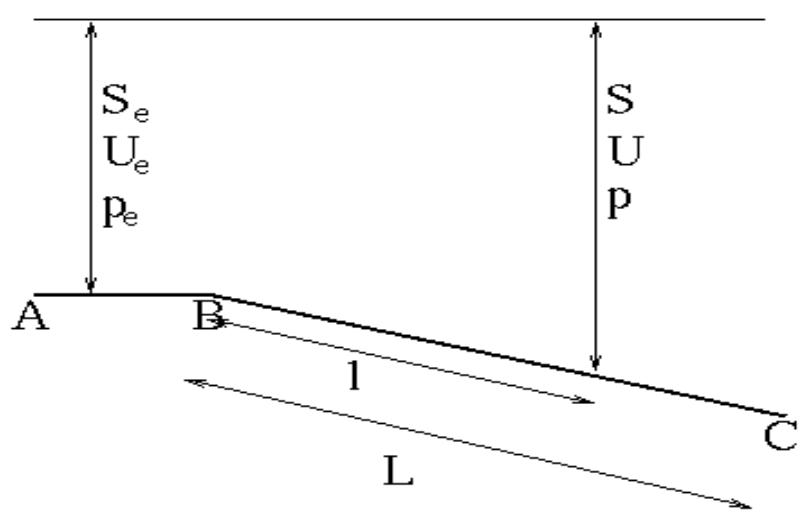

Figure 3. Ideal case for the upward thrust.

that is to say:

$$
U=\frac{U_{e} S_{e}}{S_{e}+l \sin \alpha} .
$$

Using Bernouilli's theorem, $p$ can be derived as a function of the inflow pressure $p_{e}$ :

$$
p=p_{e}+\frac{1}{2} \rho U_{e}^{2}\left[1-\left(\frac{S_{e}}{S_{e}+l \sin \alpha}\right)^{2}\right] .
$$

After integration in (3), we get:

$$
\begin{aligned}
T_{i d} & \approx\left(T_{i d}\right)_{1}+\left(T_{i d}\right)_{2}, \quad \text { with: } \\
\left(T_{i d}\right)_{1} & =L p_{e}, \\
\left(T_{i d}\right)_{2} & =\frac{L \rho U_{e}^{2}}{2}\left[1-\frac{1}{1+\frac{L \sin \alpha}{S_{e}}}\right] .
\end{aligned}
$$

$\left(T_{i d}\right)_{1}$ is function of $L$ and $p_{e}$. In fact, the transformation of the kinetic energy into a pressure force because of the deceleration of the flow is quantified by the $\left(T_{i d}\right)_{2}$ contribution, and that's why we focuss on this second part of the ideal force $T_{i d}$. The control's efficiency is then evaluated by the pressure recovery coefficient $C_{r}$, defined by:

$$
C_{r}=100 \frac{T_{\text {num }}-\left(T_{i d}\right)_{1}}{\left(T_{i d}\right)_{2}} .
$$

$T_{\text {num }}$ is numerically evaluated with $(3) .\left(T_{i d}\right)_{1}$ and $\left(T_{i d}\right)_{2}$ are computed by the expressions $(4)$ and $(5)$, with $p_{e}$ and $\rho$ beeing respectively the numerical inflow pressure and density, and $U_{e}=U_{\infty}$.

The taller $C_{r}$ is, the more efficient the control is.

\subsection{Description of the uncontrolled flow}

Without any control, the flow exhibits a periodic behaviour. Vortices are created from point B, and after some merging phenomena are conveyed downstream towards the outflow boundary, where they leave the computational domain. The isolines of several quantities such as the pressure, the vorticity, the Coherent Structures 
isolated with the Weiss criterion [2] and the stream function are displayed at three different times of a period $T_{400}$ (Fig. 4). For further informations, the accurate description of the flow's dynamics has been carefully explained [13]. In this present work, the important quantity to take care of is the $C_{r}$ evolution as a function of the time. As it will be shown in the following results, without any control the temporal average value of $C_{r}$ is equal to $64.6 \%$. The control processes will so have to increase this reference value.
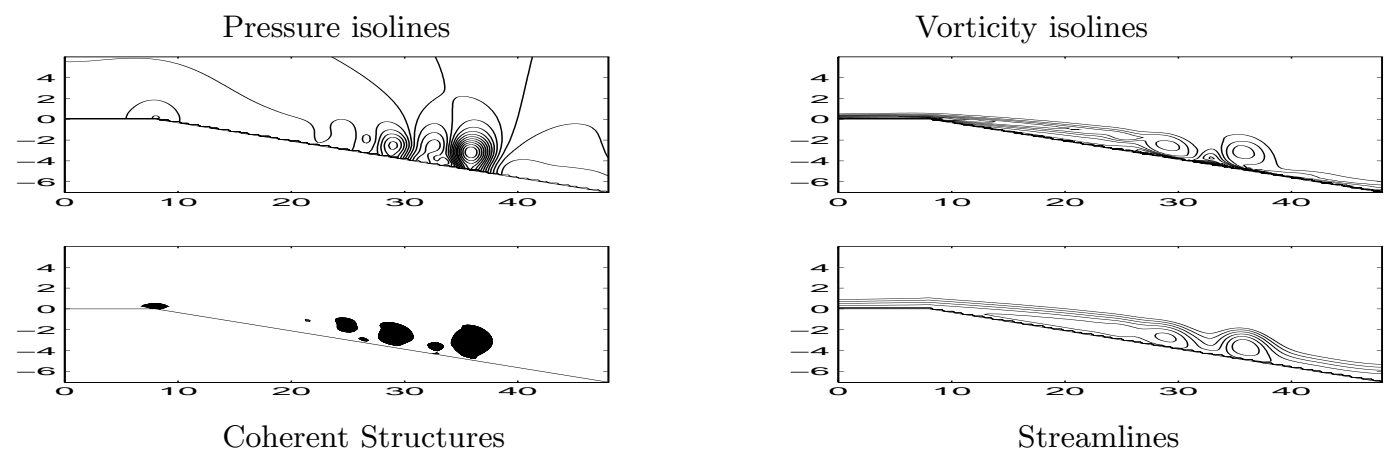

Coherent Structures
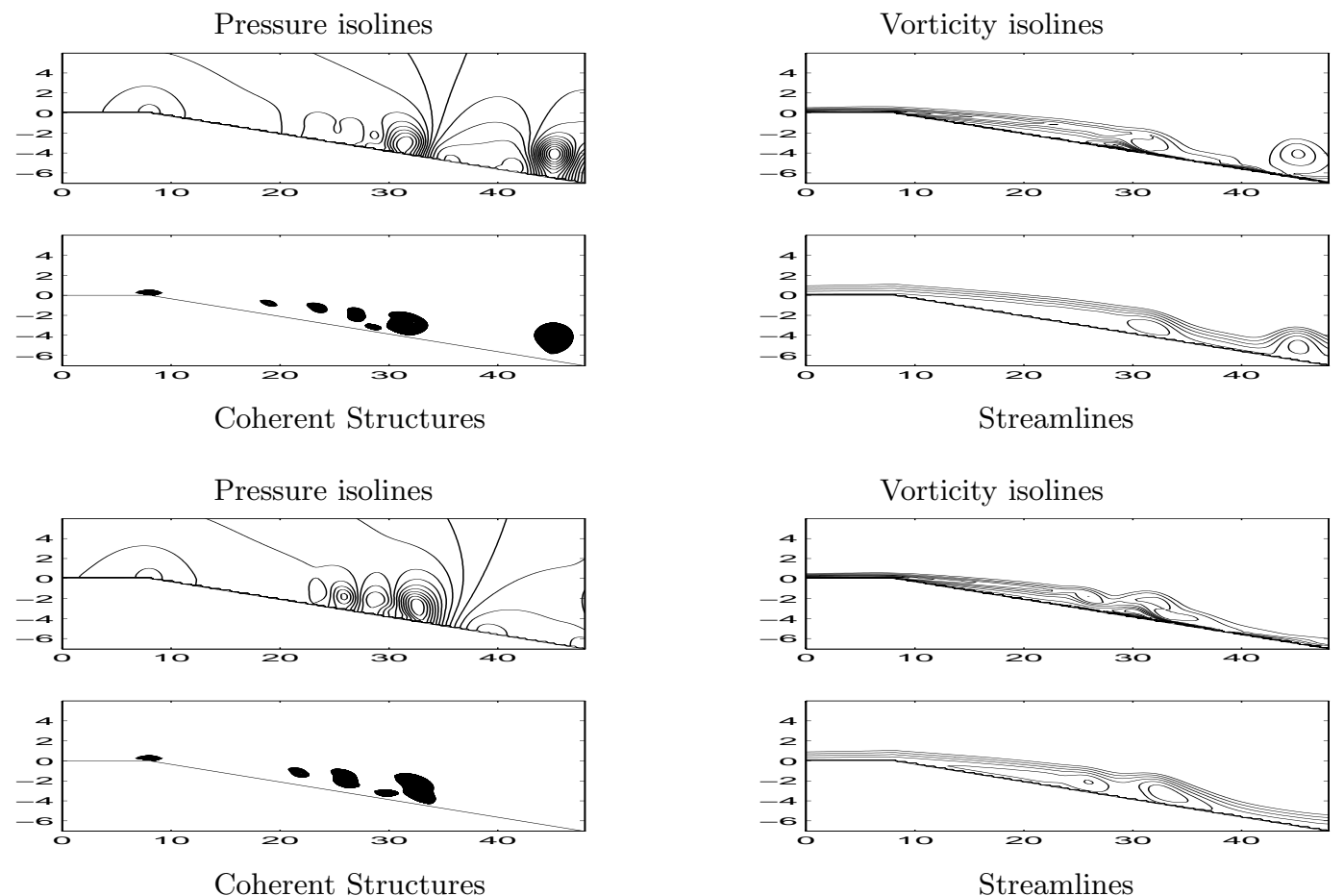

FigurE 4. $R e_{\delta}=400 . t=T_{400}$ (top), $t=T_{400}+2 T_{400} / 5$ (middle), $t=T_{400}+4 T_{400} / 5$ (bottom). 


\section{Active CONTROL}

\subsection{The closed loop control}

We first remark that at any time of the uncontrolled simulation, the location of the vortices is closely connected to the tangent pressure gradient (or the normal vorticity gradient) on the dihedral boundary (Fig. 5). This property was also checked both experimentally [18] and numerically [23]. This property is used to guess
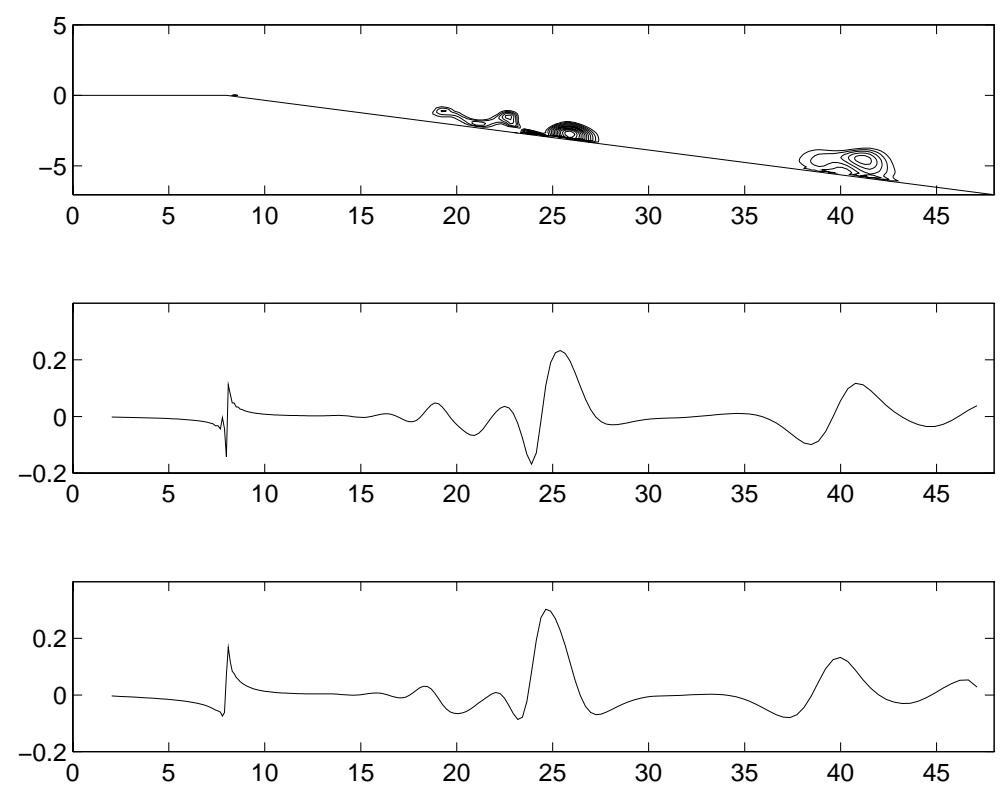

Figure 5. Correlation between the vortices location (top) and the values of $\frac{1}{\rho} \frac{\partial p}{\partial \tau}$ (middle) or $-\frac{1}{R e} \frac{\partial \omega}{\partial n}$ (bottom) on the dihedral.

the vortices location by analysing the tangent pressure gradient profile on the wall. As a consequence, a rather simple active control technique can be suggested, like Hernandez did in a close configuration [16], which is called "closed loop control".

Let us consider an intrusive sensor at point S (Fig. 6). The tangent pressure gradient on the dihedral at node $\mathrm{S},\left(\frac{\partial p}{\partial \tau}\right)_{S}$, is measured at each time step of the simulation. Then, a Vortex Generator Jet at node V, with a thickness e, is induced by specifying a vertical velocity whose amplitude $q_{V}(t)$ is given by:

$$
q_{V}(t)=\varepsilon \frac{\left(\frac{\partial p}{\partial \tau}\right)_{S}^{\prime}(t)}{\left[\left(\frac{\partial p}{\partial \tau}\right)_{S}^{\prime}\right]_{\max }}
$$

$\left(\frac{\partial p}{\partial \tau}\right)_{S}^{\prime}(t)$ is the tangent pressure gradient variation around the temporal average value of $\left(\frac{\partial p}{\partial \tau}\right)(t)$, regularly updated during the simulation. $\left[\left(\frac{\partial p}{\partial \tau}\right)_{S}^{\prime}\right]_{\max }$ is the tallest value of $\left|\left(\frac{\partial p}{\partial \tau}\right)_{S}^{\prime}(t)\right|$, recorded during a previous uncontrolled simulation. Finally, $\varepsilon=0.0025$ is the amplification coefficient. Here, the transfer function is 


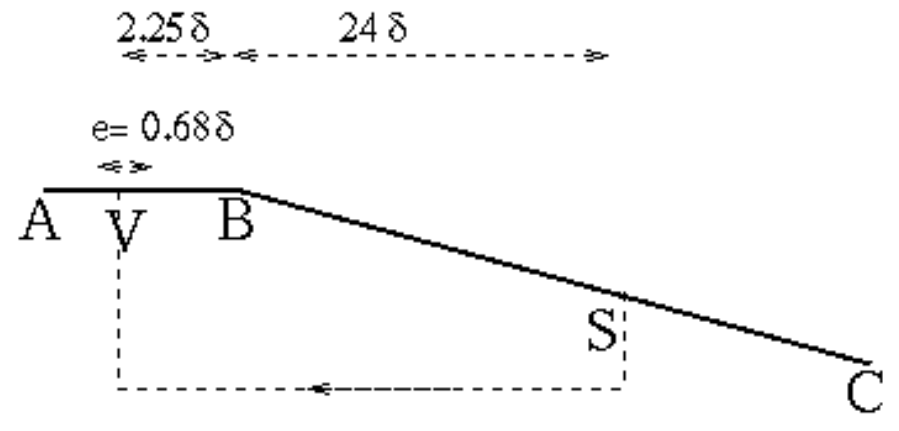

Figure 6. Closed loop control.

very simple, and made exclusively of an amplifier to supply a signal whose level is hoped sufficient to excite the system. Provided that the informations recorded at the sensor make a report on the frequency of the instabilities responsible for the low value of $C_{r}$, we can hope that the jet generated by the actuator will significantly change the flow characteristics and perhaps increase the $C_{r}$ coefficient, since it will be pulsed at a relevant frequency.

The control begins at $t=900$. After a transitional regime, the signal $q_{V}(t)$ becomes periodic, and its amplitude is in the order of $4.5 \%$ of the velocity $U_{\infty}$ (Fig. 7a). It should be noticed that the temporal average value of the signal is equal to zero. Several observations can be performed on the controlled solution.

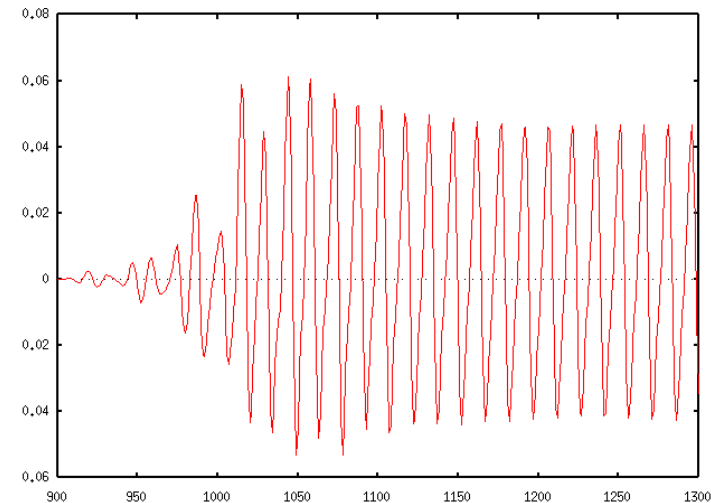

(a) $q_{V}(t)$

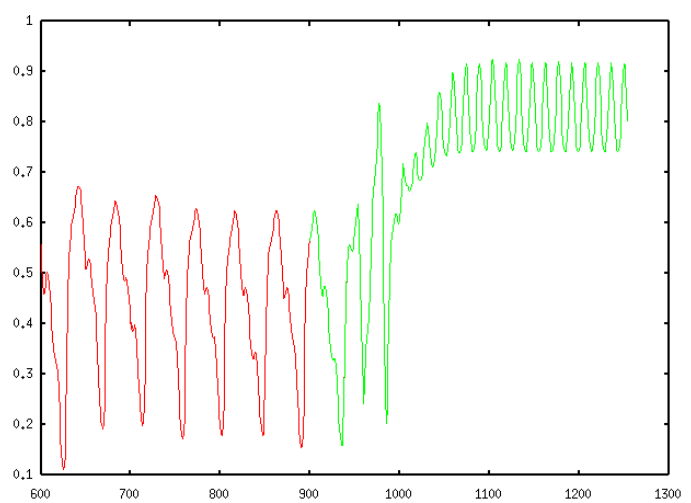

(b) $u(t)$ at a given point in the flow

FiguRE 7. $q_{V}(t)$, and $u(t)$ at a given point in the flow.

First, the fundamental frequency of the flow has been multiplied by a factor 3. For example, we can see the horizontal velocity signal as a function of the time at a given point in the flow in the zone where vortices evolve (Fig. 7b). The amplitude of the fluctuations is also smaller for the controlled flow than for the uncontrolled one. Then, the temporal average recirculation area, defined as the zone where the horizontal velocity is negative, has been drastically reduced (Fig. 8). Moreover, vortices are smaller. They do not interact together anymore, and are directly conveyed towards the outflow (Fig. 9). Finally, the average value of the $C_{r}$ coefficient has been increased up to $84.3 \%$, which confirms that this control procedure is efficient (Fig. 10).

These results are preserved provided that the actuator $\mathrm{V}$ is located next to point $\mathrm{B}$ (just upstream or just downstream), and provided that the sensor $\mathrm{S}$ is far enough from point $\mathrm{B}$, in the zone where vortices are already created and are more or less kindly conveyed towards the outflow boundary. Other numerical tests show that the addition of a phase displacement in the transfer function (6) did not change the results. An accurate description of the influence of each of the previous parameters such as the thickness of the jet, the $\varepsilon$ value, the distance of the sensor and of the actuator to point $\mathrm{B}$ will be found in [12]. 


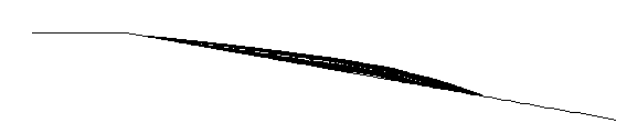

Uncontrolled average recirculation area

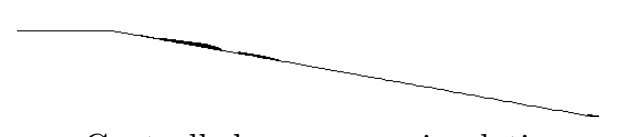

Controlled average recirculation area.

Figure 8. Average recirculation areas.

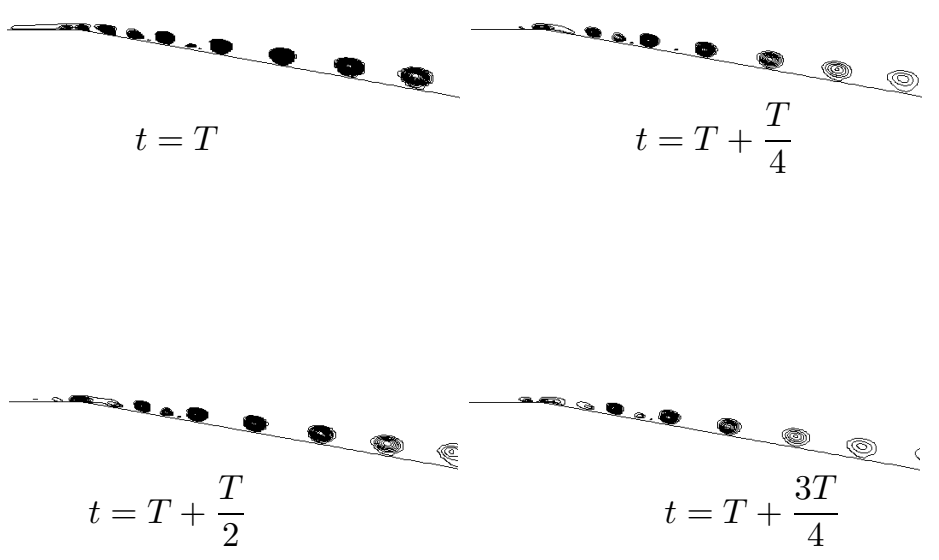

Figure 9. Coherent Structures with closed loop control during a period $T$.

These observations allow us to conclude that the closed loop control process is actually efficient, because the relevant characteristic frequency of the instabilities has been catched by the sensor, and suitably reflected to the actuator. It generates an important recirculation area reduction, and as a consequence leads to a better pressure recovery coefficient.

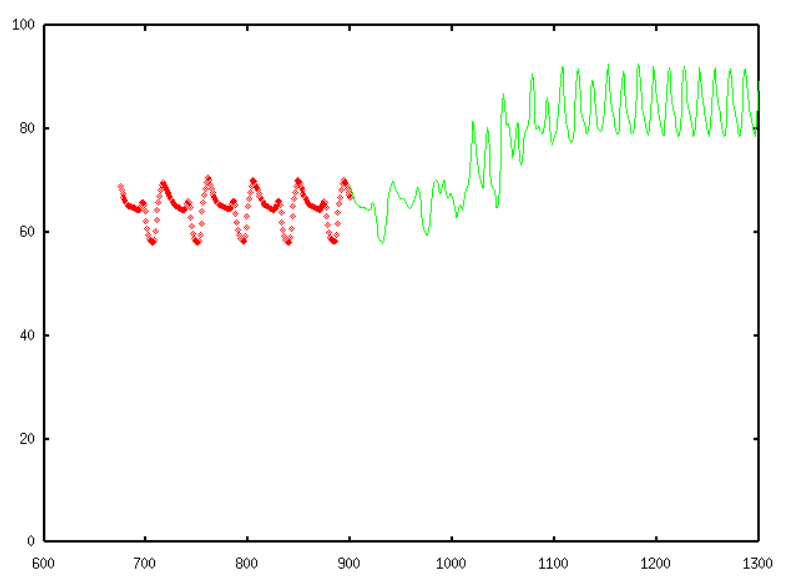

Figure 10. $C_{r}=f(t)$ (The control procedure is applied at $t=900$ ). 


\subsection{The adaptative control}

In the previous control, the transfer function is rather simple, and has been found by a fairly intuitive way. Now, we wonder if it would be possible to improve it, by using an algorithm which could progressively learn by himself the link between the $\left(\frac{\partial p}{\partial \tau}\right)_{S}(t)$ value as a function of some previous velocities induced at the actuator $\mathrm{V}$. The transfer function will continuously have to identify the system, in order to find the suitable control to produce. Nevertheless, the physical aspect as well as the governing equations of the phenomena will not be taken into account. A detailed explanation of the principle of these methods will be found in [17]. In fact, an adaptative system is a system whose effect is adjustable such that its behaviour and its efficiency get better and better during the time with regard to an identified criterion, because of its environment. Then, it would be perhaps possible to specify a given vertical velocity at node $\mathrm{V}$ and at time $t$ as a function of a desired future $\left(\frac{\partial p}{\partial \tau}\right)_{S}$ value at node $\mathrm{S}$. As a consequence, we are first looking for the $n p$ real weights $w_{k}^{i},(1 \leq i \leq n p)$, which would check as better as possible:

$$
\left(\frac{\partial p}{\partial \tau}\right)_{S}^{k} \approx \sum_{i=1}^{n p} w_{k}^{i} q_{V}^{k-r-i}
$$

$r$ is a delay parameter. $\left(\frac{\partial p}{\partial \tau}\right)_{S}^{k}$ is the tangent pressure gradient at the sensor $\mathrm{S}$ and time $t_{k}$. $q_{V}^{k-r-i}$ is the vertical velocity imposed at node $\mathrm{V}$ and time $t_{k-r-i}$, and $w_{k}^{i}(1 \leq i \leq n p)$ are the $n p$ unknown we are looking for.

The $r$ coefficient is numerically deduced by the time needed on the uncontrolled flow for a vertical velocity induced at the actuator $\mathrm{V}$ to have repercussions recorded at the sensor $\mathrm{S}$. We choose here $n p=7$. The $w_{k}^{i}$ values $(1 \leq i \leq n p)$ are regularly updated with the use of the Last Mean Square algorithm [26] on the previous closed loop simulation, until the convergence is reached. Then, the control procedure can begin parallel to the continuously updating of the weights. Using (7), we can deduce the quantity $q_{V}^{k}$ to impose as a function of a desired $\left(\frac{\partial p}{\partial \tau}\right)_{S}^{k+r+1}$ value which is noted $D_{S}^{k+r+1}$ :

$$
q_{V}^{k}=\frac{D_{S}^{k+r+1}-\sum_{i=2}^{n p} w_{k+r+1}^{i} q_{V}^{k+1-i}}{w_{k+r+1}^{1}} .
$$

Of course, at time $t_{k}$, the $w_{k+r+1}^{i}(1 \leq i \leq n p)$ values are not available. That's why we have to assume that $w_{k+r+1}^{i} \approx w_{k}^{i}$, that is to say that the $w_{k}^{i}$ values $(1 \leq i \leq n p)$ are slowly varying with regard to the time. Finally, we get:

$$
q_{V}^{k}=\frac{D_{S}^{k+r+1}-\sum_{i=2}^{n p} w_{k}^{i} q_{V}^{k+1-i}}{w_{k}^{1}}
$$

A relation such as (8) does not ensure that the vertical velocity $q_{V}^{k}$ remains bounded. As a consequence, a troncature procedure has to be added to avoid $\left|q_{V}^{k}\right|$ beeing greater than $0.045 U_{\infty}$.

Finally, the $D_{S}^{k+r+1}$ value is chosen as:

$$
D_{S}^{k+r+1}=\beta \sum_{i=1}^{n p} w_{k}^{i} q_{V}^{k-r-i}+(1-\beta)\left(\overline{\frac{\partial p}{\partial \tau}}\right)_{S},
$$



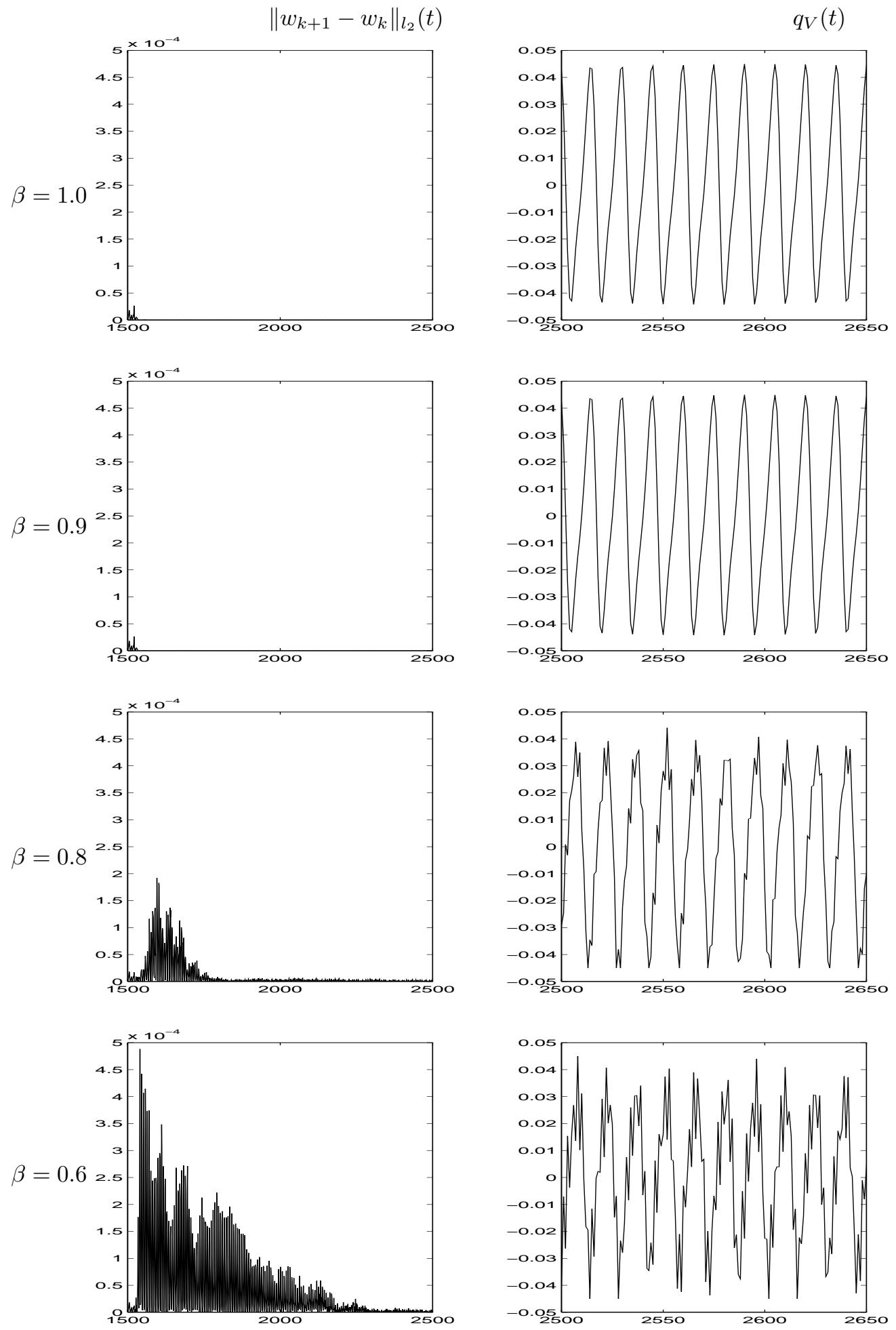

Figure 11. Adaptative control as a function of $\beta$. $\left\|w_{k+1}-w_{k}\right\|_{l_{2}}(t)$ (left) and $q_{V}(t)$ (right). 
where $\left(\frac{\overline{\partial p}}{\partial \tau}\right)_{S}$ is the average tangent pressure gradient at node $\mathrm{S}$, regularly updated during the controlled simulation. We have to choose $\beta$ between $\beta=0$ and $\beta=1$. Of course, the case $\beta=1$ would lead exactly to the same results as the closed loop control ones.

Remark. For the previous closed loop control, the actuations were updated at each numerical time step $\delta t$ imposed by the CFL condition. For the adaptative control, the actuations are updated at each $\Delta t=T_{400} / 45$, where $T_{400}$ is the fundamental frequency of the uncontrolled simulation. Of course, in order to make the comparisons possible, we checked than even with the actuations updated at each $\Delta t$, the closed loop control gives the same results as the ones previously displayed.

The initial datum used is a flow obtained with the closed loop control. Results are analysed for different values of $\beta$. On Figure 11, we can see the evolution of $\left\|w_{k+1}-w_{k}\right\|_{l^{2}}(t)$ from the start of the control process until the established controlled regime is reached (left row), as well as the evolution of $q_{V}(t)$ when the established controlled regime is reached (right row). First, we can see that the smaller $\beta$ is, the more complex $q_{V}(t)$ is, and the longer it is for the vector $w_{k}$ to reach the convergence.

Nevertheless, the $C_{r}$ coefficient is nearly the same as the one obtained with the closed loop control (Tab. 1). No significant improvement is observed. So, we can deduce that even if the transfer function seems more sophisticated, the main fact which makes such a control efficient is the vortices frequency crossing which is recorded at the sensor $\mathrm{S}$, and has repercussions on the $q_{V}(t)$ signal.

TABLE 1. Adaptative control $C_{r}$ as a function of $\beta$.

\begin{tabular}{|c|c|}
\hline$\beta$ value & $C_{r}$ \\
\hline$\beta=1.0$ & $84.3 \%$ \\
\hline$\beta=0.9$ & $84.7 \%$ \\
\hline$\beta=0.8$ & $84.4 \%$ \\
\hline$\beta=0.6$ & $83.5 \%$ \\
\hline
\end{tabular}

\subsection{The physical ramp control}

Now, we would like to use several actuators and sensors, to see if it could bring higher efficiency. To do it, we consider a control process based on physical arguments. Obviously, the success of such a method strongly depends on the physical phenomena's understanding capability. To take an example, a reference work was performed to decrease the drag generated by a turbulent boundary layer on a wall [9]. The idea was to use Vortex Generator Jets to move away from the wall the tangential viscous strains, responsible for the drag. Then, a normal wall velocity was imposed as a function of the normal velocity at a given distance just upon the wall. Results were rather good, and an interpretation of these good performances was given later [15]. Unfortunately, with the use of intrusive sensors in the wall, results were not so good.

We propose now a recent control type based on the Lighthill theory [20]. This control has been recently used by Koumoutsakos in a different configuration [19].

\subsubsection{Basis principle}

The generation of vorticity near a no-slip wall caused by a VGJ can be decomposed in two successive steps. In the first one, the fluid is considered inviscid. If we assume that a jet is produced by an actuator located at a point $A_{1}^{\prime}$ on the wall during an elementary time step $\Delta t$, since the fluid is a non viscous one, a slip velocity $U_{g}$ is created on the wall, whose modulus at a given point $S_{1}$ on the wall is inversely proportionnal to the distance $S_{1} A_{1}^{\prime}$ (Fig. 12). In the second step, this fictitious slip velocity is canceled by the vorticity normal flux generation 


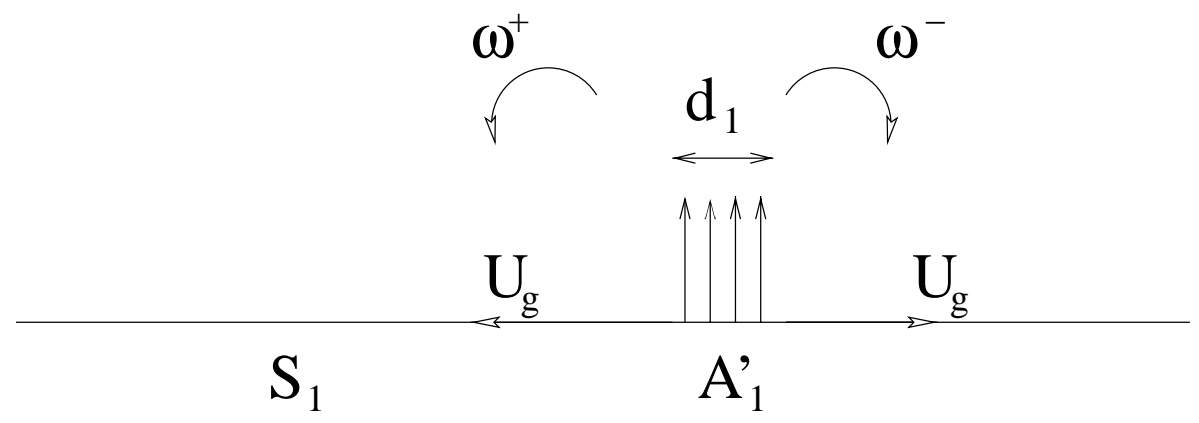

Figure 12. Physical ramp control: basis principle.

$\frac{\partial \omega}{\partial y}$, in order to ensure the actual no-slip boundary condition. As a consequence, the generated circulation in the flow on each elementary segment of the wall whose lenght is $\delta s$ is then $\delta \Gamma=U_{g} \delta s$, and we have:

$$
\delta \Gamma=\nu \frac{\partial \omega}{\partial y} \Delta t \delta s
$$

$\nu=\mu / \rho_{r}$ is the kinematic viscosity of the fluid. Finally, a vertical VGJ centered on $A_{1}^{\prime}$, whose thickness is $d_{1}$ and whose amplitude is $q_{1}\left(q_{1}>0\right.$ corresponding to blowing and $q_{1}<0$ corresponding to suction), generated during an elementary time step $\Delta t$ produces at node $S_{1}$ a vorticity normal flux defined by:

$$
\frac{\partial \omega}{\partial y}\left(x_{1}\right)=\mathrm{d} s \frac{q_{1}}{2 \pi \nu \Delta t} \int_{-d_{1} / 2}^{d_{1} / 2} \frac{\mathrm{d} s}{x_{1}-x_{1}^{\prime}} .
$$

Considering now $N$ actuators $A_{j}^{\prime}(1 \leq j \leq N)$, and $N$ sensors $S_{i}(1 \leq i \leq N)$, the normal flux of vorticity produced on $S_{i}$ because of the jets generated at the $N$ actuators during an elementary time step $\Delta t$ can be deduced by superimposition:

$$
\frac{\partial \omega}{\partial y}\left(x_{i}\right)=\frac{1}{2 \pi \nu \Delta t} \sum_{j=1}^{N} q_{j} \mathrm{~d} s \int_{-d_{j} / 2}^{d_{j} / 2} \frac{\mathrm{d} s}{x_{i}-x_{j}^{\prime}-s}
$$

Since the flow is a slightly compressible one, we can admit, in a first approximation, that $\nabla \cdot \vec{u}=0$. Using the incompressible Navier-Stokes equation, as well as the fact that on a no-slip wall we have $\vec{u}=\frac{\partial \vec{u}}{\partial t}=\overrightarrow{0}$, we can easily prove:

$$
-\nu\left(\frac{\partial \omega}{\partial y}\right)=\frac{1}{\rho}\left(\frac{\partial p}{\partial x}\right)
$$

Then, the relation (9) becomes:

$$
\frac{\partial p}{\partial x}\left(x_{i}\right)=-\frac{\rho}{2 \pi \Delta t} \sum_{j=1}^{N} q_{j} \int_{-d_{j} / 2}^{d_{j} / 2} \frac{\mathrm{d} s}{x_{i}-x_{j}^{\prime}-s}
$$

\subsubsection{Control procedure}

Now, we consider the set of $N$ sensors $S_{i}(1 \leq i \leq N)$ and actuators $A_{j}(1 \leq j \leq N)$, alternately allocated on the dihedral between the points $\mathrm{B}$ and $\mathrm{C}$ (Fig. 13). With the previous analysis, it is now possible to establish 


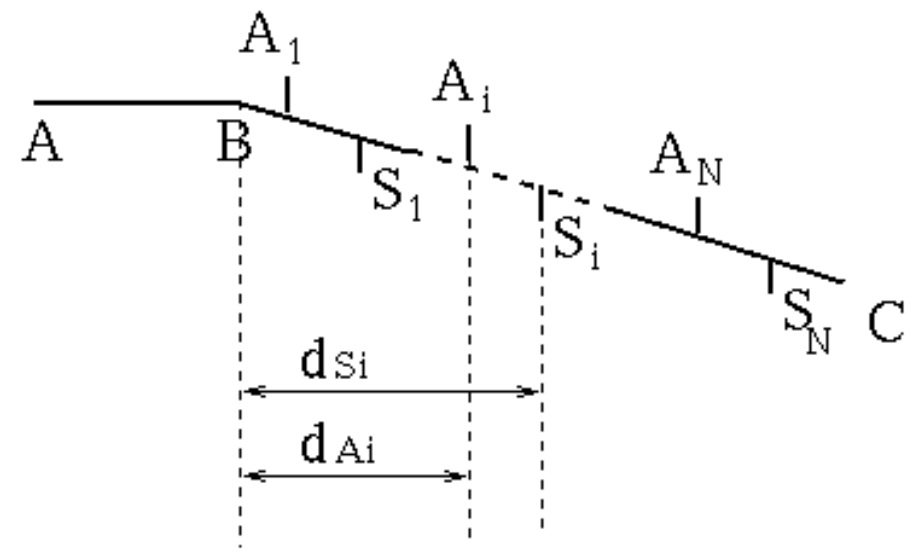

Figure 13. Physical ramp control: sensors and actuators locations.

explicitly the amplitude of the actuation vector $Q^{k}$ to impose at the actuators in order to achieve a desired pressure tangential gradient vector $D^{k}$ at the sensors at time $t^{k}$ :

$$
Q^{k}=B^{-1}\left(D^{k}-X^{k-1}\right)
$$

with:

$$
\begin{aligned}
Q^{k} & =\left[q_{1}^{k}\left(x_{1}^{\prime}\right), q_{2}^{k}\left(x_{2}^{\prime}\right), \ldots, q_{N}^{k}\left(x_{N}^{\prime}\right)\right]^{T}, \\
X^{k-1} & =-\left[\frac{1}{\rho^{k-1}} \frac{\partial p^{k-1}}{\partial \tau}\left(x_{1}\right), \frac{1}{\rho^{k-1}} \frac{\partial p^{k-1}}{\partial \tau}\left(x_{2}\right), \ldots, \frac{1}{\rho^{k-1}} \frac{\partial p^{k-1}}{\partial \tau}\left(x_{N}\right)\right]^{T}, \\
B_{i j} & =\frac{1}{2 \pi} \ln \left|\frac{x_{j}^{\prime}-x_{i}-d_{j} / 2}{x_{j}^{\prime}-x_{i}+d_{j} / 2}\right|, \quad 1 \leq i \leq N, 1 \leq j \leq N .
\end{aligned}
$$

Here, we choose $D^{k}$ to be equal to the vector whose component $i$ is the average value of $\frac{1}{\rho} \frac{\partial p}{\partial \tau}\left(x_{i}\right)$ regularly updated since the begining of the simulation $(1 \leq i \leq N)$.

Numerically speaking, we actually use the relation (11) in a modified formulation:

$$
Q^{k}=\varepsilon_{K} B^{-1}\left(D^{k}-X^{k-1}\right)
$$

$\varepsilon_{K}$ is a parameter which has to be adjusted. In order to do it, we simply remark that the closed loop control can be considered as a physical ramp control with one sensor and one actuator. As a consequence, the closed loop control results obtained with the actuator just downstream the point B are used to derive the value of $\varepsilon_{K}$ in the physical ramp control with one sensor and one actuator which leads to these same results.

Remark. It would have been also possible, with such a control, to use $M$ sensors and $N$ actuators, with $M<N$, and to add $N-M$ additional constraints in order to make the $B$ matrix inversible. For example, we could take $M=N-1$, and ask to the total mass flux through the wall to be equal to zero. It would be specified, 

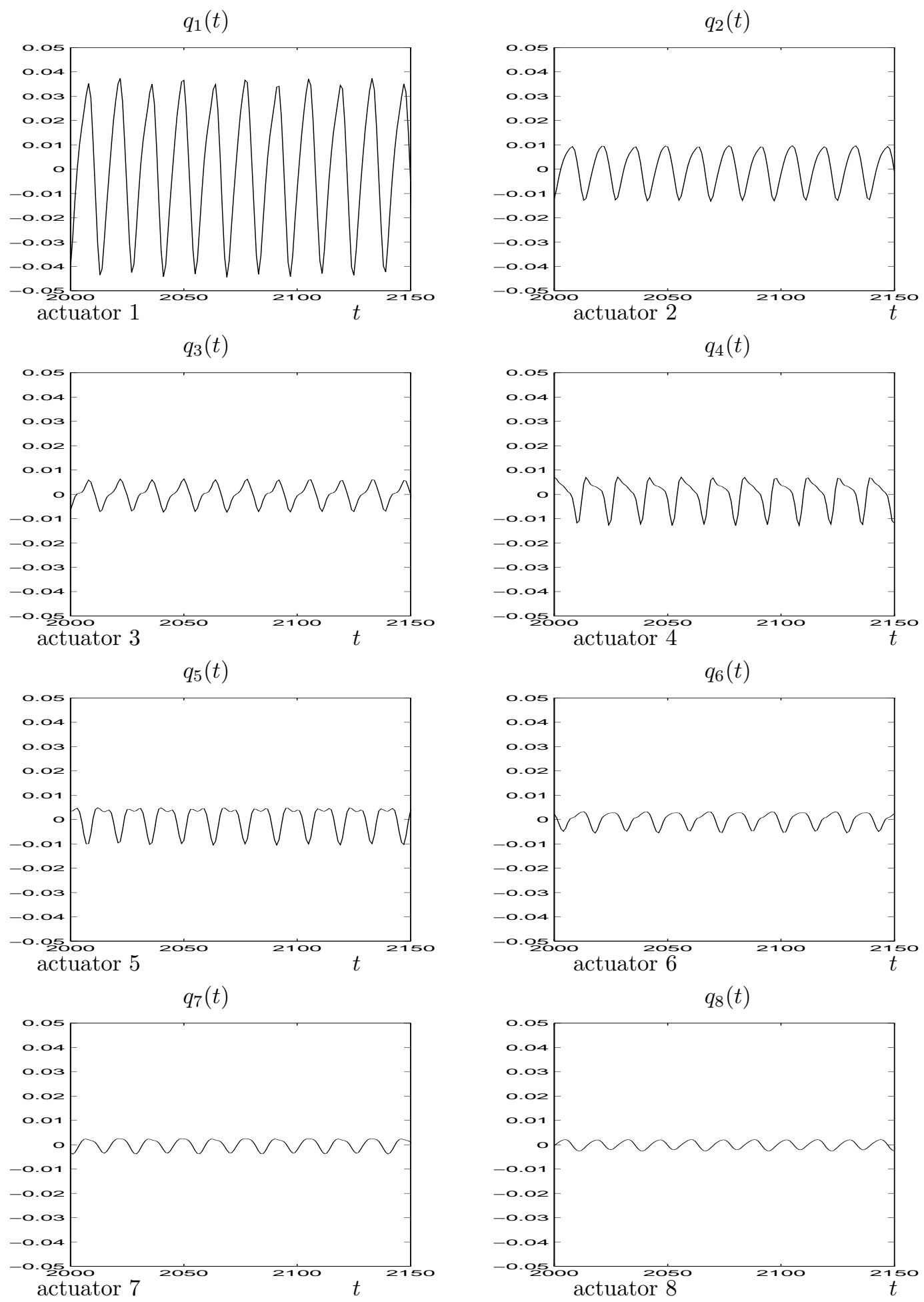

Figure 14. Physical ramp control: actuations $Q(t)$ for $N=8$. 

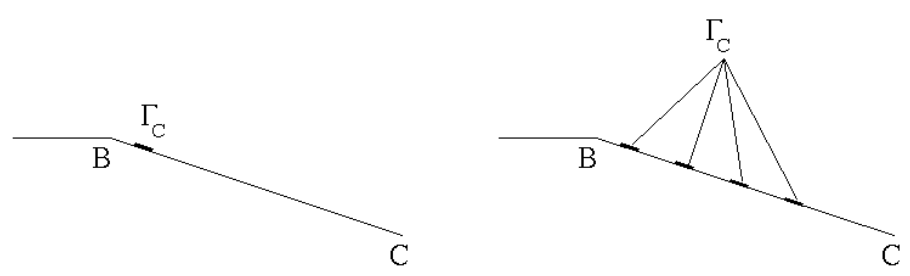

Figure 15. Sub-optimal control: definition of $\Gamma_{C}$. Local (left) or distribuated (right).

provided that the density $\rho$ is considered as constant and that all the actuators have the same thickness, by:

$$
\sum_{j=1}^{N} q_{j}^{k}=0 .
$$

Results are presented for $N=8$. Table 2 gives the sensors and actuators location on the dihedral. Then, the

TABLE 2. $d_{S_{i}}$ and $d_{A_{i}}$ for $n=8$.

\begin{tabular}{|c|c|c|c|c|}
\hline Locations & 1 & 2 & 3 & 4 \\
\hline Sensors $d_{S_{i}}$ & $12,5 \delta_{200}$ & $16,5 \delta_{200}$ & $21,5 \delta_{200}$ & $26,5 \delta_{200}$ \\
\hline Actuators $d_{A_{i}}$ & $9 \delta_{200}$ & $14 \delta_{200}$ & $19 \delta_{200}$ & $24 \delta_{200}$ \\
\hline \hline Locations & 5 & 6 & 7 & 8 \\
\hline Sensors $d_{S_{i}}$ & $31,5 \delta_{200}$ & $36,5 \delta_{200}$ & $41,5 \delta_{200}$ & $46,5 \delta_{200}$ \\
\hline Actuators $d_{A_{i}}$ & $29 \delta_{200}$ & $34 \delta_{200}$ & $39 \delta_{200}$ & $44 \delta_{200}$ \\
\hline
\end{tabular}

Figure 14 gives the evolution of $q_{j}^{k}(t)(1 \leq j \leq 8)$ when the established controlled regime is reached. We can see that the fundamental frequency of the signal is the same whatever actuator is concerned. Moreover, the closer the actuator is to point $\mathrm{B}$, the taller the amplitude of the actuation is.

Finally, it appears that the average $C_{r}$ coefficient is about $85.9 \%$, which is an interessant improvement with regard to the two previous control procedures, since it corresponds to a relative saving of $1.9 \%$.

\subsection{The sub-optimal control}

Now, we would like to consider a control procedure based on a mathematical analysis of the governing equations: the optimal control. The governing equations are now used to conceive the control strategy. It leads to a systematic method to find retroactive control laws for the more efficient control velocities distribution in order to reach a wanted effect. This control strategy was introduced by Abergel and Temam for fluid mechanics problems [1] following the traces of Lions [21]. It was then developped by Choi et al. for the stochastic Burger equation [10]. More recently, Bewley et al. considered this control procedure in several configurations [3-6, 25], and Hernandez began to consider it for the configuration we are dealing with [16].

Let us note $\Gamma_{C}$ a part of the dihedral on which the control $\Phi$ will be applied. $\Gamma_{C}$ can be local, or distributed along the wall (Fig. 15). We then note $\Gamma_{P}=[\mathrm{BC}]$. We hope the aim of the control to be reached by minimizing the functionnal $J_{T}(\Phi, p)$ given by:

$$
J_{T}(\Phi, p)=\int_{[0, T]} \frac{\alpha}{2} \int_{\Gamma_{C}} \Phi^{2} \mathrm{~d} l+\frac{1}{2} \int_{\Gamma_{P}}\left(p-p^{i d}\right)^{2} \mathrm{~d} l \mathrm{~d} t .
$$


Such an objective is very difficult because of the very large computational time needed, as we will see later. As a consequence, the control procedure is simplified by introducing the sub-optimal control, which consists in minimizing the functionnal $J(\Phi, p)$ between two successive time steps given by:

$$
J(\Phi, p)=\frac{\alpha}{2} \int_{\Gamma_{C}} \Phi^{2} \mathrm{~d} l+\frac{1}{2} \int_{\Gamma_{P}}\left(p-p^{i d}\right)^{2} \mathrm{~d} l=J_{C}(\Phi)+J_{P}(\Phi) .
$$

$J_{C}(\Phi)$ corresponds to the cost of the control. $J_{P}(\Phi)$ permits to know how far the pressure distribution along $\Gamma_{P}$ is from an ideal distribution $p^{i d}$, which has to be specified. The greater $\alpha$ is, the more expensive is the control process.

Let us consider that the solution $U^{n}$ is given on the whole domain. We want to derive $\Phi$ to be applied between the times $t^{n}$ and $t^{n+1}$, in order to minimize the functionnal $J(\Phi, p)$ at time $t^{n+1}$ by solving:

- compressible Navier-Stokes equations from $t^{n}$ to $t^{n+1}$;

- on the dihedral,

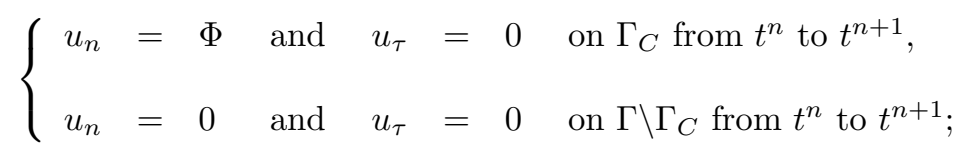

- other boundary conditions unchanged.

In order to do it, we have to explicitly express the Frechet derivative of $J(\Phi, p)$ with regard to $\Phi$. It will then be possible to make use of a descent algorithm to reach the relevant control value $\Phi$ to apply. We first have:

$$
\frac{D J}{D \Phi}(\Phi, p) \tilde{\Phi}=\alpha \int_{\Gamma_{C}} \Phi \tilde{\Phi} \mathrm{d} l+\int_{\Gamma_{P}}\left(p-p^{i d}\right) \psi \mathrm{d} l
$$

with:

$$
\psi=\frac{D p}{D \Phi} \tilde{\Phi}
$$

Since for our configuration the fluid is slightly compressible, we do the incompressiblity approximation. That's why we introduce the incompressible Navier-Stokes equations in order to derive the $u^{n+1}, v^{n+1}$ and $p^{n+1}$ values at each node of the mesh. Moreover, we consider a pressure implicit discretization:

$$
\left\{\begin{aligned}
u^{n+1}-u^{n}+\Delta t \frac{\partial p^{n+1}}{\partial x_{1}} & =R_{1}^{n}, \\
v^{n+1}-v^{n}+\Delta t \frac{\partial p^{n+1}}{\partial x_{2}} & =R_{2}^{n} \\
\frac{\partial u^{n+1}}{\partial x_{1}}+\frac{\partial v^{n+1}}{\partial x_{2}} & =0 \\
u_{n}=\Phi & \text { and } \quad u_{\tau}=0 \text { on } \Gamma_{C} \text { from } t^{n} \text { to } t^{n+1}, \\
u_{n}=0 & \text { and } \quad u_{\tau}=0 \text { on } \Gamma \backslash \Gamma_{C} \text { from } t^{n} \text { to } t^{n+1} .
\end{aligned}\right.
$$


$R_{1}^{n}$ and $R_{2}^{n}$ are residuals depending on $U^{n}$ only. The Frechet derivative with regard to $\Phi$ of this previous system takes the formulation:

$$
\left\{\begin{array}{rll}
\eta_{1}+\Delta t \frac{\partial \Psi}{\partial x_{1}} \quad= & 0 \\
\eta_{2}+\Delta t \frac{\partial \Psi}{\partial x_{2}} & =0 \\
\frac{\partial \eta_{1}}{\partial x_{1}}+\frac{\partial \eta_{2}}{\partial x_{2}} & =\quad 0 \\
\eta_{n}=\tilde{\Phi} & \text { and } \quad \eta_{\tau}=0 \text { on } \Gamma_{C} \text { from } t^{n} \text { to } t^{n+1} \\
\eta_{n}=0 & \text { and } \quad \eta_{\tau}=0 \text { on } \Gamma \backslash \Gamma_{C} \text { from } t^{n} \text { to } t^{n+1}
\end{array}\right.
$$

Here,

$$
\eta_{1}=\frac{D u^{n+1}}{D \Phi} \tilde{\Phi} \quad ; \quad \eta_{2}=\frac{D v^{n+1}}{D \Phi} \tilde{\Phi}
$$

The adjoint system can be then deduced by the scalar product with the dual variables $\left(\zeta_{1}, \zeta_{2}, \pi\right)$ :

$$
\begin{gathered}
\int_{\Omega}\left[\left(\eta_{i}+\Delta t \frac{\partial \Psi}{\partial x_{i}}\right) \zeta_{i}+\frac{\partial \eta_{i}}{\partial x_{i}} \pi\right] \mathrm{d} V=0 \\
\Longleftrightarrow \int_{\Omega}\left[\eta_{i} \zeta_{i}+\Delta t \frac{\partial\left(\Psi \zeta_{i}\right)}{\partial x_{i}}-\Delta t \Psi \frac{\partial \zeta_{i}}{\partial x_{i}}+\left(\frac{\partial\left(\eta_{i} \pi\right)}{\partial x_{i}}-\eta_{i} \frac{\partial \pi}{\partial x_{i}}\right)\right] \mathrm{d} V=0 \\
\Longleftrightarrow \int_{\Omega}\left[\eta_{i}\left(\zeta_{i}-\frac{\partial \pi}{\partial x_{i}}\right)-\Delta t \Psi \frac{\partial \zeta_{i}}{\partial x_{i}}\right] \mathrm{d} V+\int_{\partial \Omega}\left(\Delta t \Psi \zeta_{n}+\eta_{n} \pi\right) \mathrm{d} l=0 .
\end{gathered}
$$

As a consequence, the adjoint state equation is given by:

$$
\left\{\begin{array}{l}
\zeta_{i}-\frac{\partial \pi}{\partial x_{i}}=0 \\
\frac{\partial \zeta_{i}}{\partial x_{i}}=0
\end{array}\right.
$$

and the boundary condition has to be chosen in a convenient way to reach what we are looking for, that is to say an explicit expression of $\frac{D J}{D \Phi}(\Phi, p)$. To do it, we make the choice:

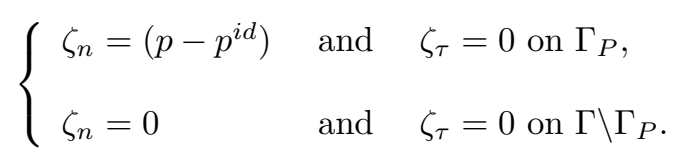


We obtain:

$$
\begin{aligned}
\int_{\partial \Omega} \Delta t \Psi \zeta_{n} \mathrm{~d} l+\int_{\partial \Omega} \eta_{n} \pi \mathrm{d} l & =0 \\
\Longleftrightarrow \quad \int_{\Gamma_{P}} \Delta t\left(p-p^{i d}\right) \frac{D p}{D \Phi} \tilde{\Phi} \mathrm{d} l+\int_{\Gamma_{C}} \pi \tilde{\Phi} \mathrm{d} l & =0 \\
\Longleftrightarrow \quad \Delta t\left(\frac{D J}{D \Phi}(\Phi, p) \tilde{\Phi}-\alpha \int_{\Gamma_{C}} \Phi \tilde{\Phi} \mathrm{d} l\right)+\int_{\Gamma_{C}} \pi \tilde{\Phi} \mathrm{d} l & =0 . \\
\frac{D J}{D \Phi}(\Phi, p) \tilde{\Phi} & =\int_{\Gamma_{C}}\left(\alpha \Phi-\frac{\pi}{\Delta t}\right) \tilde{\Phi} \mathrm{d} l .
\end{aligned}
$$

Finally, we express explicitly the Frechet derivative of $J(\Phi, p)$ as a function of the adjoint pressure $\pi$ :

$$
\frac{D J}{D \Phi}(\Phi, p)=\alpha \Phi-\frac{\pi}{\Delta t} .
$$

Remark. If $\left(\zeta_{1}, \zeta_{2}, \pi\right)$ is solution of the adjoint problem, then $\left(\zeta_{1}, \zeta_{2}, \pi+C\right)$ is also solution $(C \in \mathbb{R})$. In order to choose $C$, we set at the top left corner of the domain $\pi=0$. This choice is justified in order to ensure that $\Phi \equiv 0$ in the case where $p \equiv p^{i d}$ on $\Gamma_{P}$. Indeed, in this case we would have in the same time:

$$
\frac{D J}{D \Phi} \tilde{\Phi}=\alpha \int_{\Gamma_{C}} \Phi \tilde{\Phi} \mathrm{d} l \quad \text { and } \quad \frac{D J}{D \Phi} \tilde{\Phi}=\int_{\Gamma_{C}}\left(\alpha \Phi-\frac{\pi}{\Delta t}\right) \tilde{\Phi} \mathrm{d} l .
$$

If $p \equiv p^{i d}$ on $\Gamma_{P}$, the resolution of the adjoint problem leads to $\pi \equiv$ constante in the whole domain, then $\pi \equiv 0$ since $\pi=0$ on $\Gamma_{C}$.

The previous analysis leads to a control procedure which is summarized on the next page. Of course, the sub-optimal control is very computationaly expensive, since we have to solve at each numerical time step and at several times an adjoint problem. That's why, after the theoritical approximations we have already done, we must do now numerical approximations. The first one consists in doing only one iteration for the control updating (in fact, the adjoint problem is resolved only one time at each time step). It can be justified by the fact that since the CFL condition is very restrictive, we can consider the control to be produced at time $t^{n+1}$ very close to the control produced at time $t^{n}$, which is the initial datum for the adjoint problem. The second one is that the resolution of the adjoint problem is solved by a Gauss-Seidel relaxation method cell to cell for which we do not wait for the convergence of the solution, but stop the resolution after a given a priori number of iterations. It can also be justified for the same previous reason. Even if the sub-optimal control is cheaper than the optimal control, it costs roughly $100 \%$ CPU time more, whereas all the previous controls have negligible computational costs. We set $\alpha=0.1$ and $\mu=3.75 \cdot 10^{-6}$. This last value was deduced with the help of several numerical tests.

The first results obtained with a local $\Gamma_{C}$ at the same location as the actuator used for the closed loop control are rather disappointing. Indeed, when the established controlled flow is reached, the average $C_{r}$ coefficient is equal to $70.4 \%$. The adjoint pressure on $\Gamma_{C}$ oscillates around zero (Fig. 16a), but $q_{V}(t)$ remains always negative (Fig. 16b). It is fortunately better than the uncontrolled flow, but similar results are obtained with a passive technique by imposing a permanent suction through the actuator $V$ with $q_{V}(t) \equiv-0.045 U_{\infty}$. Moreover, it should be noted that the fundamental frequency of the uncontrolled simulation is still detected in this controlled flow, contrary to the three previous control processes. A decrease of $\mu$ leads to a deterioration of the control efficiency, whereas an increase of $\mu$ prevents the $q_{V}(t)$ signal to oscillate, and compels it to stay at the maximum value permitted by the truncature procedure. If we now use a distributed $\Gamma_{C}$, at the same location as the actuators used for the physical ramp control, the average $C_{r}$ coefficient is equal to $71.8 \%$, which is not a very significant improvement, and the actuations on each actuator remain all negative. 


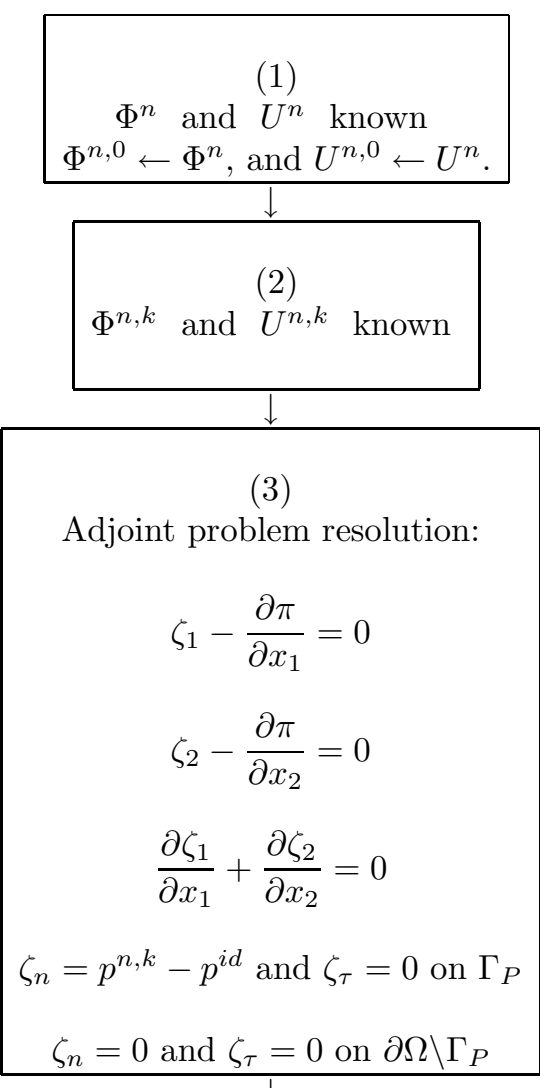

(4)

Obtention of $\Phi^{n, k+1}$ with: $\Phi^{n, k+1}=\Phi^{n, k}-\mu\left(\alpha \Phi^{n, k}-\frac{\pi}{\Delta t}\right)$

$\downarrow$

(5)

Computation of $U^{n, k+1}$ by resolution of compressible Navier-Stokes equations, with: $\Phi=\Phi^{n, k+1}$

(6)

If $\Phi^{n, k}$ has converged, then we go at step (7) If not, we go at step (2) to do an additional iteration on $k$

(7)

$\Phi^{n+1} \leftarrow \Phi^{n, k+1}$, and $U^{n+1} \leftarrow U^{n, k+1}$. 


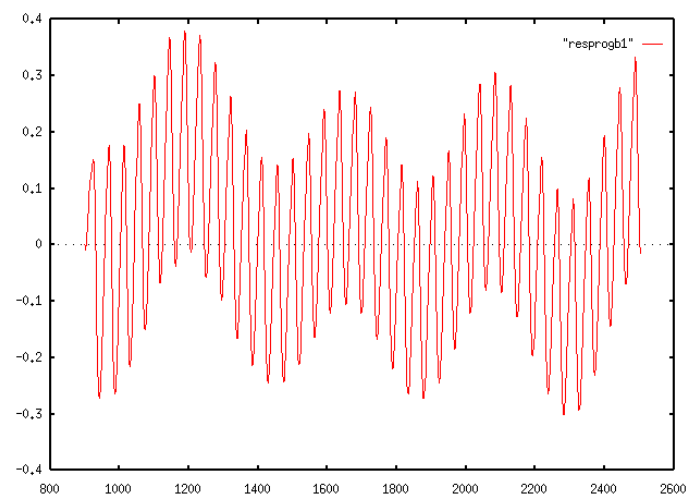

(a) $\pi(t)$ at a node of $\Gamma_{C}$.

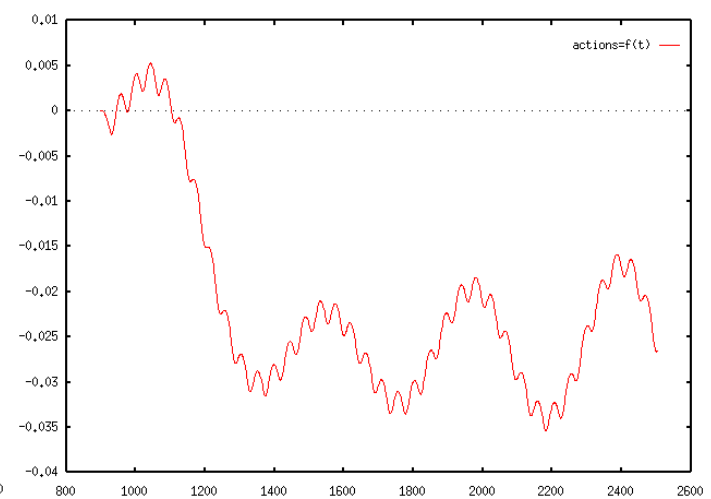

(b) $q_{V}(t)$ on $\Gamma_{C}$.

FiguRE 16. Sub-optimal control.

We so tried to use the sub-optimal control algorithm with a local $\Gamma_{C}$ but also a local $\Gamma_{P}$. The unique difference is that to solve the adjoint problem, the non homogeneous boundary condition is imposed only on a small part of $[B C]$, corresponding to the location of the sensor for the closed loop control. This way to proceed allows us to recover a $C_{r}$ coefficient of $82.3 \%$, which is close to the results obtained with the previous controls. the signal $q_{V}(t)$ is this time a crenel type signal because of the truncated procedure (Fig. 17). Of course, even if it is numerically satisfactory, the use of this local $\Gamma_{P}$ makes the previous theoritical analysis no more valid.

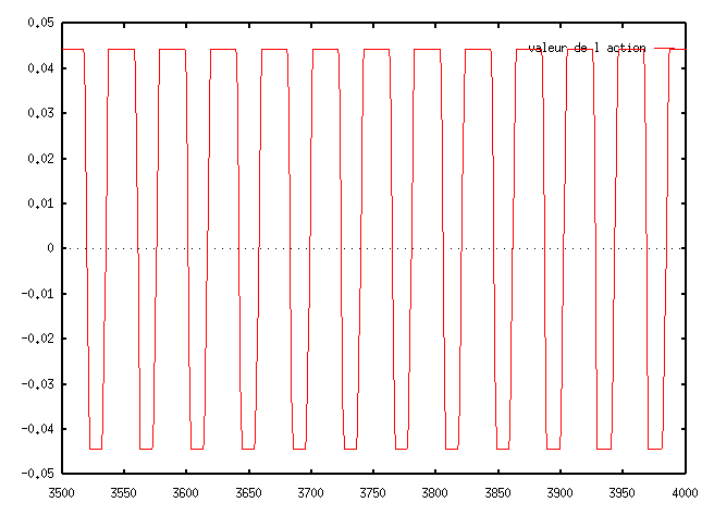

Figure 17. $q_{V}(t)$ on $\Gamma_{C}$, sub-optimal control.

Use of a local $\Gamma_{P}$ for the resoultion of the adjoint problem.

These observations can be interpretated by the fact that with the use of a global $\Gamma_{P}$, the vortices passage frequency is no more detected. Indeed, there are continually several vortices on $\Gamma_{P}$. As a consequence, the adjoint pressure on $\Gamma_{C}$ is computed from this global average flow on $\Gamma_{P}$, and does not vary fastly enough. On the contrary, when $\Gamma_{P}$ is local, the adjoint pressure on $\Gamma_{C}$ takes better into account the highest frequencies corresponding to the vortices passage on $\Gamma_{P}$.

\section{Conclusion}

In this paper, four active control techniques are compared to increase the upward thrust on a dihedral plane, generated by a compressible viscous flow. This upward thrust is essentially induced by the pressure on the 
dihedral since the evolution is laminar, and is characterized by the value of a defined pressure recovery coefficient $C_{r}$. In order to do it, several tangential pressure gradient sensors as well as several Vortex Actuator Jets are used on the wall.

The closed loop control, whose transfer function simply consists in an amplification, reaches a remarkable result by improving the $C_{r}$ coefficient from $64.6 \%$ to $84.3 \%$. It is due to the fact that the actuator is excited at a frequency corresponding to the instabilities frequency detected at the sensor location. This control cancels the vortices interactions, and significantly decreases the temporal average recirculation area.

With the same sensor and the same actuator, the adaptative control is used to modify the transfer function. The principle is to find and to use a correlation law between the velocities induced at the actuator and the signal recorded at the sensor. This control carries out at the same time both a training phase and a control phase. Unfortunately, no significant improvement was noticed with regard to the closed loop control results. In fact, since the jet at the actuator remained at the same fundamental frequency and at the same amplitude, results obtained are nearly the same.

A third control strategy is based on physical arguments, and developped in previous works by Koumoutsakos. We applied it to our configuration by using 8 sensors and 8 actuators. The quantization of a vorticity normal flux produced in the vicinity of a normal jet generated on a no slip wall is given by a mechanical analysis of the phenomena. Then, after a generalization to several actuators, the amplitudes of the 8 jets to impose on the dihedral in order to reach a desired discret pressure tangent flux at the 8 sensors are deduced. This way to proceed leads to an increase of $C_{r}$ in the order of $1.9 \%$ with regard to the previous controls. In fact, not only the relevant frequency is produced by the jets (which ensures at least the same results as the closed loop ones), but the pressure field is also locally modified closed to each of the 8 actuators, what permits to improve also a little the results.

Finally, the last control procedure is based on the governing equations of the flow. The aim is to minimize a functionnal which quantifies how far the pressure distribution along the downstream part of the dihedral is from a desired pressure distribution. Because of the slightly compressible nature of the flow, incompressible Navier-Stokes equations are used in a first approximation. Consequently, an adjoint problem is considered and resolved, to obtain the expression of the derivative of this functionnal to use a descent type algorithm. In a first time, results are rather disappointing, since the pressure recovery coefficient $C_{r}$ is equal to $70.4 \%$. Nevertheless, in a second time, the fact of using only a reduced part of the downstream part of the dihedral to specify the boundary conditions for the resolution of the adjoint problem leads to results very close to those obtained by the previous control methods. This can be certainly explained by the fact that the use of the whole downstream part of the dihedral for the resolution of the adjoint problem prevents the high frequencies to be specified at the actuator, because of an average process. On the contrary, the use of a small part of the downstream part of the dihedral allows the high frequencies to be specified to the actuator.

The author wishes to thank particularly C.H. Bruneau for fruitful discussions about several topics developped in this work.

\section{REFERENCES}

[1] F. Abergel and R. Temam, On some control problems in fluid mechanics. Theoret. Comput. Fluid Dynamics 1 (1990) 303.

[2] C. Basdevant and T. Philipovitch, On the "Weiss criterion" in two-dimensional turbulence. Physica D (1994) 17-34.

[3] T. Bewley, H. Choi, R. Temam and P. Moin, Optimal feedback control of turbulent channel flow. Technical report annual research briefs, center for turbulence research (1993).

[4] T. Bewley and P. Moin, Optimal control of turbulent channel flow. ASMEDE 75 (1994).

[5] T. Bewley, P. Moin and R. Temam. A method for optimizing feedback control rules for wall-bounded turbulent flows based on control theory', Forum on control of transitional and turbulent flows, ASME fluids engineering conference (1996).

[6] T.R. Bewley, P. Moin and R. Temam, Optimal and robust approaches for linear and non linear regulation problems in fluid mechanics, in 28th AIAA fluid dynamics conference, 4th AIAA shear flow control conference (1997). 
[7] C.H. Bruneau and E. Creusé, Towards a transparent boundary condition for the compressible Navier-Stokes equations. Internat. J. Numer. Methods Fluids (to appear).

[8] H. Choi, P. Moin and J. Kim, Direct numerical simulation of turbulent flow over riblets. J. Fluid Mech. 255 (1993) 503-539.

[9] H. Choi, P. Moin and J. Kim, Active turbulence control for drag reduction in wall bounded flow. J. Fluid Mech. 262 (1994) 75-110.

[10] H. Choi, R. Temam, P. Moin and J. Kim, Feedback control for unsteady flow and its application to the stockastic Burgers equation. J. Fluid Mech. 253 (1993) 509-543.

[11] J. Cousteix, Couche limite laminaire. Cepadues Editions (1988).

[12] E. Creusé, Simulation et contrôle actif d'écoulements compressibles, Thèse de Doctorat. Université Bordeaux I (2000).

[13] E. Creusé and I. Mortazavi, Vortex dynamics over a dihedral plane in a transitional slightly compressible flow: A computational study. European J. Mech. B Fluids (to appear).

[14] L. Fezoui, S. Lanteri, B. Larrouturou and C. Olivier, Résolution numérique des équations de Navier-Stokes pour un fluide compressible en maillage triangulaire. Rapport de recherche INRIA 1033 (1989).

[15] E.P. Hammond, T.R. Bewley and P. Moin, Observed mechanisms for turbulence attenuation and enhancement in oppositioncontrolled wall bounded flow, Technical Report. Department of Mechanical Engineering, Stanford University, California (1998).

[16] G. Hernandez, Contrôle actif des instabilités hydrodynamiques des écoulements subsoniques compressibles, Ph.D. Thesis. CERFACS, France (1996).

[17] R. Insermann, K.H. Lachmann and D. Matko, Adaptive control system. Prentice Hall, New-York (1992).

[18] A.V. Johansson, P.H. Alfredsson and J. Kim, Evolution and dynamics of shear-layer structures in near-wall turbulence. $J$. Fluid Mech. 224 (1991) 579-599.

[19] P. Koumoutsakos, Active control of vortex-wall interactions. Phys. Fluids 9 (1997) 3808-3816.

[20] M.J. Lighthill, Boundary layer theory. Introduction. J. Rosenhead, Oxford University Press, New-York (1963).

[21] J.L. Lions, Contrôle optimal des systèmes gouvernés par des équations aux dérivées partielles. Dunod, Paris (1969).

[22] P. Moin and T. Bewley, Feedback control of turbulence. Appl. Mech. Rev 47 (1994).

[23] P. Moin and J. Kim, The structure of vorticity fields in turbulent channel flows. Part 1: Analysis of instantaneous field and statistical correlations. J. Fluid Mech. 155 (1985).

[24] T.J. Poinsot and S.K. Lele, Boundary conditions for direct simulations of compressible viscous flows. J. Comput. Phys. 101 (1992) 104-129.

[25] R. Temam, T. Bewley and P. Moin, Control of turbulent flows, in 18th IFIP TC7 conferences on system modelling and optimisation. Detroit, Michigan (1997).

[26] B. Widrow and S.D. Stearns, Adaptive signal processing. Prentice-Hall (1985). 